\title{
Numerical Simulations of the Lunar Penetrating Radar and Investigations of the Geological Structures of the Lunar Regolith Layer at the Chang'E 3 Landing Site
}

\author{
Chunyu Ding, ${ }^{1,2,3}$ Yan Su, ${ }^{1,2}$ Shuguo Xing, ${ }^{1,2}$ Shun Dai, ${ }^{1,2}$ Yuan Xiao, ${ }^{1,2,3}$ Jianqing Feng, \\ Danqing Liu, ${ }^{4}$ and Chunlai $\mathrm{Li}^{1,2}$
}

${ }^{1}$ Key Laboratory of Lunar and Deep Space Exploration, Chinese Academy of Sciences, Beijing 100012, China
${ }^{2}$ National Astronomical Observatories, Chinese Academy of Sciences, Beijing 100012, China
${ }^{3}$ University of Chinese Academy of Sciences, Beijing 100049, China
${ }^{4}$ Renmin University of China, Beijing 100872, China

Correspondence should be addressed to Yan Su; suyan@nao.cas.cn

Received 21 December 2016; Revised 12 March 2017; Accepted 3 April 2017; Published 23 May 2017

Academic Editor: Han Guo

Copyright (C) 2017 Chunyu Ding et al. This is an open access article distributed under the Creative Commons Attribution License, which permits unrestricted use, distribution, and reproduction in any medium, provided the original work is properly cited.

In the process of lunar exploration, and specifically when studying lunar surface structure and thickness, the established lunar regolith model is usually a uniform and ideal structural model, which is not well-suited to describe the real structure of the lunar regolith layer. The present study aims to explain the geological structural information contained in the channel 2 LPR (lunar penetrating radar) data. In this paper, the random medium theory and Apollo drilling core data are used to construct a modeling method based on discrete heterogeneous random media, and the simulation data are processed and collected by the electromagnetic numerical method FDTD (finite-difference time domain). When comparing the LPR data with the simulated data, the heterogeneous random medium model is more consistent with the actual distribution of the media in the lunar regolith layer. It is indicated that the interior structure of the lunar regolith layer at the landing site is not a pure lunar regolith medium but rather a regolith-rock mixture, with rocks of different sizes and shapes. Finally, several reasons are given to explain the formation of the geological structures of the lunar regolith layer at the Chang'E 3 landing site, as well as the possible geological stratification structure.

\section{Introduction}

The exploration of the internal structure of moon has been ongoing since the first time a human being landed on the surface of the moon in the 1960s. According to the analysis of seismic data, the internal structure of the moon can be roughly divided into a lunar crust, lunar mantle, and lunar nucleus. The thickness of the outermost lunar shell is approximately $60 \sim 65 \mathrm{~km}$, the top $1 \sim 2 \mathrm{~km}$ of which mainly consists of lunar regolith and rock fragments [1]. On December 2, 2013, China successfully launched the Chang'E 3 spacecraft to explore the moon. The LPR was one of the important payloads on the Chang'E 3. As a high-resolution lunar surface penetrating radar, LPR consists of two channels.
The first channel is centered at a $60 \mathrm{MHz}$ frequency and has a meter-level resolution in simulated lunar rock material. It is used to detect the subsurface lunar structures along the path of the Yutu rover. The second channel is centered at a $500 \mathrm{MHz}$ frequency, with a resolution of less than $30 \mathrm{~cm}$ in the simulated lunar regolith, and it is used to detect the internal structure of the lunar regolith and its thickness. ALSE (Apollo Lunar Sounder Experiment), LRS (Lunar Radar Sounder), and LPR are all surface penetration radars, which are usually used to detect subsurface lunar structures. However, the LPR resolution is significantly higher than that of either ALSE or LRS. Especially for the detection of lunar regolith, the resolution of ALSE and LRS does not have sufficient detection accuracy, and the deepest drilling depth of an experiment 
TABLE 1: Basic parameters of lunar penetrating radar.

\begin{tabular}{lcc}
\hline LPR parameters & Ch. 1 & Ch. 2 \\
\hline Center frequency (MHz) & 60 & 500 \\
Pulse emission voltage (V) & 1000 & 400 \\
Bandwidth (MHz) & $40-80$ & $250-750$ \\
Sampling interval (ns) & 2.5 & 0.3125 \\
Range resolution & Meter level & $<30 \mathrm{~cm}$ \\
Detection depth (m) & $\geqq 100 \mathrm{~m}$ & $\leqq 30 \mathrm{~m}$ \\
\hline
\end{tabular}

with a sample return is $294.5 \mathrm{~cm}$ from Apollo 17 [2]. However, it is obvious that the above drilling depths cannot reach the bottom of the lunar regolith layer in the lunar maria, where the average layer depth is approximately $5 \mathrm{~m} \mathrm{[3].}$ Therefore, the LPR data are valuable for the study of the internal structure of the lunar regolith. Furthermore, the core diameter from the Apollo borehole is no more than $4 \mathrm{~cm}$, which means that no data, other than that of lunar penetrating radar, can directly verify that there exist rock fragments with diameters larger than $4 \mathrm{~cm}$ in the lunar regolith.

The study of the internal structure or thickness of the lunar regolith requires theoretical modeling, regardless of whether active radar detection or passive microwave radiometer detection is used. For instance, Shkuratov and Bondarenko [3] established a simplified ideal uniform lunar regolith structure model to obtain the first map of the distribution of lunar regolith thickness on the front side of the moon using the data of the Arecibo Astronomical Observatory's $70 \mathrm{~cm}$-wavelength ground-based radar in combination with the iron and titanium abundances of the front side of the lunar surface. Lan and Zhang [4] assumed that the lunar regolith layer is a uniform medium in their study of the thickness of the lunar regolith using microwaves. Fa and Jin [5] assumed that the lunar regolith layer has a uniform distribution of dense particle media to simulate the bright temperature of the multichannel lunar surface radiation. Meng et al. [6] assumed that the permittivity of the lunar regolith follows changes in depth, established a nonisotropic lunar model, and analyzed lunar thickness, frequency, and other effects on the bright temperature. Using the same assumption as Meng et al., Chen et al. [7] established twolayer and three-layer models. In Chen et al's studies, the lunar surface structure was simulated using GprMax, and its waveform characteristics were analyzed.

However, the LPR data show that the internal structure of the lunar regolith is very complex and the above modeling method is too ideal, so it is difficult to describe the real structure of the lunar regolith. Therefore, here, we employ the random medium model theory, Apollo drilling sample data, and geomorphologic images to establish a heterogeneous random medium model of the lunar regolith layer. Then, the FDTD numerical method is used to simulate the propagation of the electromagnetic wave in the model. The result of the echoes is obtained and compared with the LPR data.

\section{Lunar Penetrating Radar (LPR)}

LPR is a surface penetrating radar with a carrier frequency in the nanosecond pulse time domain whose working principle [8] is as follows: the transmitter antenna emits an electromagnetic wave into the lunar subsurface; when the propagating electromagnetic wave meets a heterogeneous medium, layered interface, or other buried object, phenomena such as reflection, diffraction, and scattering occur; the receiving antenna receives echo signals, such as reflections and scatterings; by analyzing and processing the received echo signals, we will obtain information about the geological structure of the lunar regolith along the road of Yutu rover. The basic parameters of the LPR are shown in Table 1.

The echo signals are mainly affected by the electromagnetic wave propagation velocity $v$, seen in (1), and the attenuation $\alpha$, seen in (2), in the Yutu rover detection process.

$$
\begin{aligned}
& \alpha=\omega\left[\frac{\mu \varepsilon_{r}}{2}\left(\sqrt{1+\left(\frac{\sigma}{\omega \varepsilon_{r}}\right)^{2}}-1\right)\right]^{1 / 2}, \\
& v=c\left\{\frac{\varepsilon_{r}}{2 \varepsilon_{0}}\left[\left(\sqrt{1+\left(\frac{\sigma}{\omega \varepsilon_{r}}\right)^{2}}+1\right)\right]\right\}^{-1 / 2},
\end{aligned}
$$

where $\omega$ is the angular frequency, $c$ is the speed of light in free space, $\mu$ is the permeability, $\varepsilon_{r}$ is the permittivity, and $\sigma$ is the electrical conductivity. The loss tangent, $\tan \delta=\sigma / \omega \varepsilon_{r}$, reflects the loss of energy when propagating through the lunar regolith.

\section{The Heterogeneous Random Medium Model of the Lunar Regolith Layer}

As shown in Figure 1, there is a random distribution of lunar rocks of different sizes on the lunar surface. Therefore, it is inferred that there exist a large number of small-scale and irregularly distributed media, such as basalt grains and breccias, under the lunar surface. The radar echo signal is affected by these small-scale media during the detection process, which is probably the reason for the confusing radar gram. These small-scale media distributions can be considered as random processes to study the characteristics of their permittivity. The technique of random medium modeling is applied to the seismic numerical simulation [911]. 

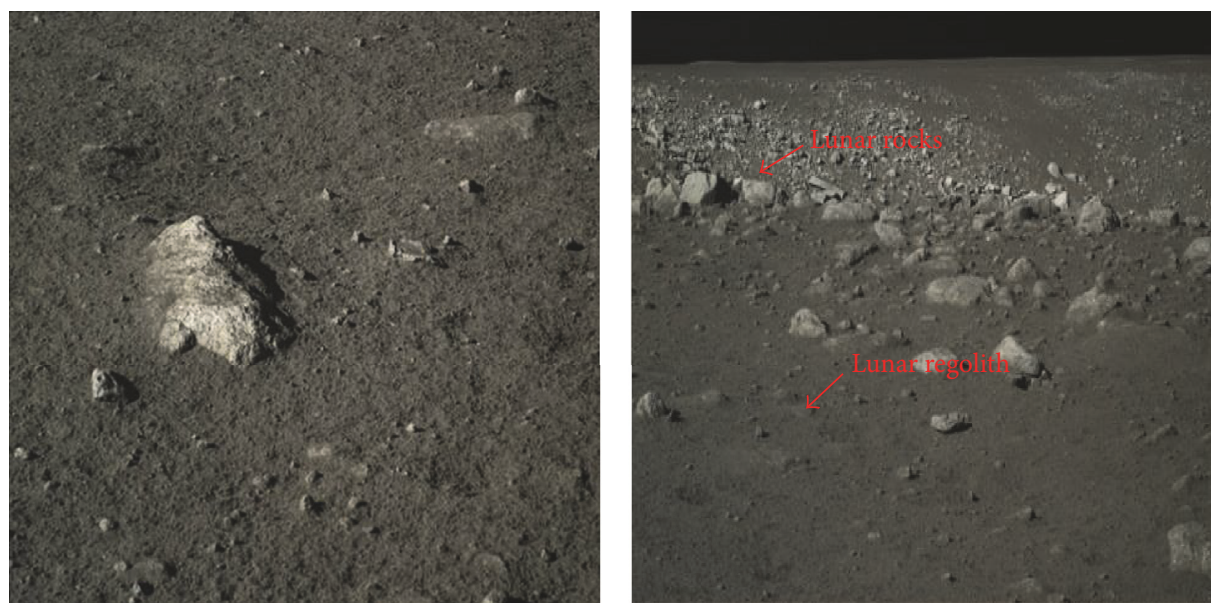

FIGURE 1: The photos were taken by the panoramic camera on the Yutu rover.

On the assumption of stationary random processes, the permittivity of the random medium model is expressed by

$$
\varepsilon(x, z)=\varepsilon_{m}+\delta(x, z) \varepsilon_{f}(x, z)
$$

where $\varepsilon_{m}$ is the mean permittivity of the background, $\delta(x, z)$ is the standard deviation, and $\varepsilon_{f}(x, z)$ is a small-scale random perturbation whose spatial distribution is subject to

$$
\begin{gathered}
\phi(x, z)=\exp \left\{-\left[\left(\frac{x \cos \theta+z \sin \theta}{a}\right)^{2}\right.\right. \\
\left.\left.+\left(\frac{-x \sin \theta+z \cos \theta}{b}\right)^{2}\right]^{1 /(1+r)}\right\},
\end{gathered}
$$

where $\phi(x, z)$ is the autocorrelation function; parameters $a$ and $b$ are the horizontal and vertical autocorrelation lengths, respectively; $\theta$ is the autocorrelation angle; and $r$ is the roughness factor at the microscale. When $r=0,(4)$ is the Gaussian autocorrelation function. When $r=1,(4)$ is the exponential autocorrelation function. When $0<r<1$, (4) is a hybrid autocorrelation function [12].

The algorithm of the established discrete random medium model is as follows.

Step 1. The power spectra density function, $\Phi\left(k_{x}, k_{z}\right)$, of the spatial random perturbation function, $\varepsilon_{f}(x, z)$, was calculated from (4) and is defined as

$$
\Phi\left(k_{x}, k_{z}\right)=\sum_{x=0}^{M-1} \sum_{z=0}^{N-1} \phi(x, z) e^{-j 2 \pi\left(k_{x} x / M+k_{z} z / N\right)} .
$$

Step 2. We used a random phase function $\varphi\left(k_{x}, k_{z}\right)$, which is an independent and evenly distributed two-dimensional random sequence on the interval $[0,2 \pi)$, to calculate the random power spectra function $W\left(k_{x}, k_{z}\right)$, which is defined as

$$
W\left(k_{x}, k_{z}\right)=\sqrt{\Phi\left(k_{x}, k_{z}\right)} \cdot e^{j \cdot \varphi\left(k_{x}, k_{z}\right)} .
$$

Step 3. We obtained the spatial perturbation function of the random medium, $\varepsilon_{f}(x, z)$, by using the inverse Fourier transform of the random power spectra function.

$$
\varepsilon_{f}(x, z)=\frac{1}{M N} \sum_{k_{x}=0}^{M-1} \sum_{k_{z}=0}^{N-1} W\left(k_{x}, k_{z}\right) e^{j 2 \pi\left(k_{x} x / M+k_{z} z / N\right)} .
$$

Step 4. The spatial perturbation function was normalized. Moreover, we substitute (7) into (3) to achieve a discrete heterogeneous random medium model.

The lunar regolith layer medium should be composed of a lunar regolith and rock mixture at the Chang'E 3 landing site area, which is shown in Figure 2. This type of lunar geological structure can be called a regolith-rocks mixture. Hence, the random medium theory modeling method is used to describe the geological structure of a regolith-rocks mixture. According to previous knowledge from the Apollo samples, the lunar regolith is mainly composed of mineral and rock fragments, breccia debris, all kinds of glass material, meteorite fragments, and so forth, with a permittivity in the range of 2.3 to 3.5 and loss tangent in the range of $0.005 \sim 0.009$. The mare region is mainly composed of basalt, with a permittivity in the range of 6.6 to 8.6 and a loss tangent in the range of $0.009 \sim 0.016$ [13]. Therefore, we assume that the dielectric properties of the random medium model of the lunar regolith range from 2.3 to 8.6 and the loss tangent ranges from 0.005 to 0.016 . A set of autocorrelation lengths, $a$ and $b$, equal to $0.05 \mathrm{~m}, 0.1 \mathrm{~m}, 0.2 \mathrm{~m}$, and $0.3 \mathrm{~m}$, are chosen to establish four discrete heterogeneous random medium models with different characteristics, which are shown in Figure 3. The model size is $5 \mathrm{~m} \times 5 \mathrm{~m}$ and the length of the discrete step is $0.01 \mathrm{~m}$. The channel 2 antenna, which is mounted on the bottom of the Yutu rover, is $0.3 \mathrm{~m}$ away from the lunar surface. Hence, we set the antenna height as $0.3 \mathrm{~m}$ off the lunar surface in the model. The first layer is the vacuum layer, with a permittivity of 1 and a depth of $0.3 \mathrm{~m}$. The second layer is the heterogeneous random medium of the lunar regolith layer with a background permittivity of 4.8 , autocorrelation angle of 0 , roughness factor of 0 , and model standard deviation of 


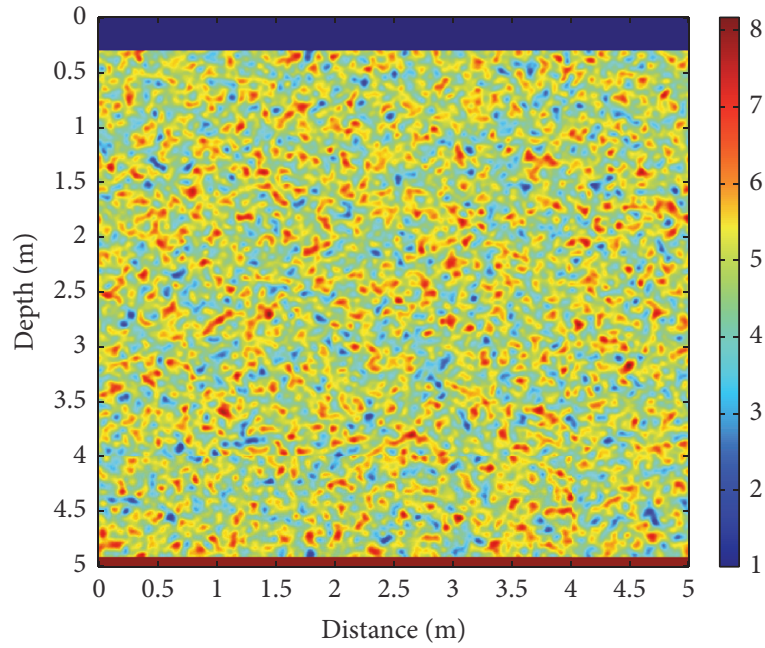

(a)

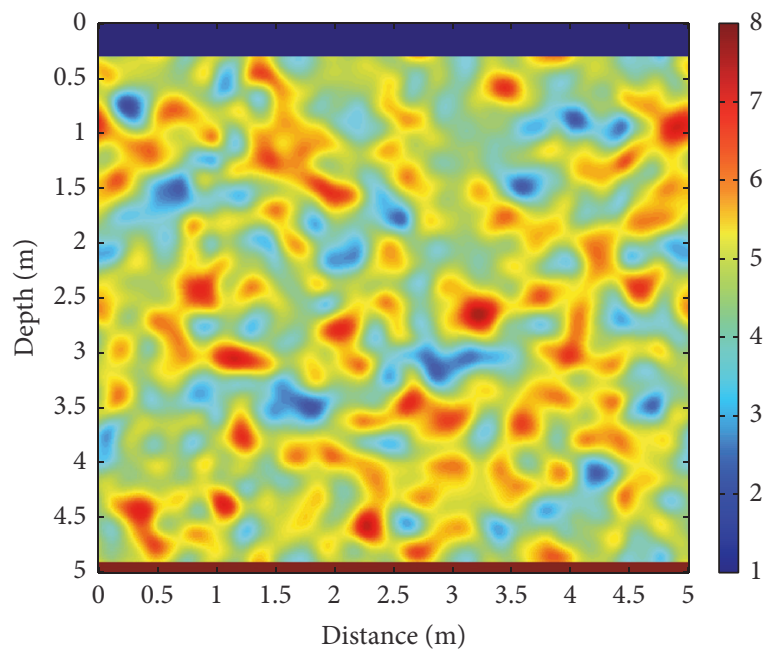

(c)

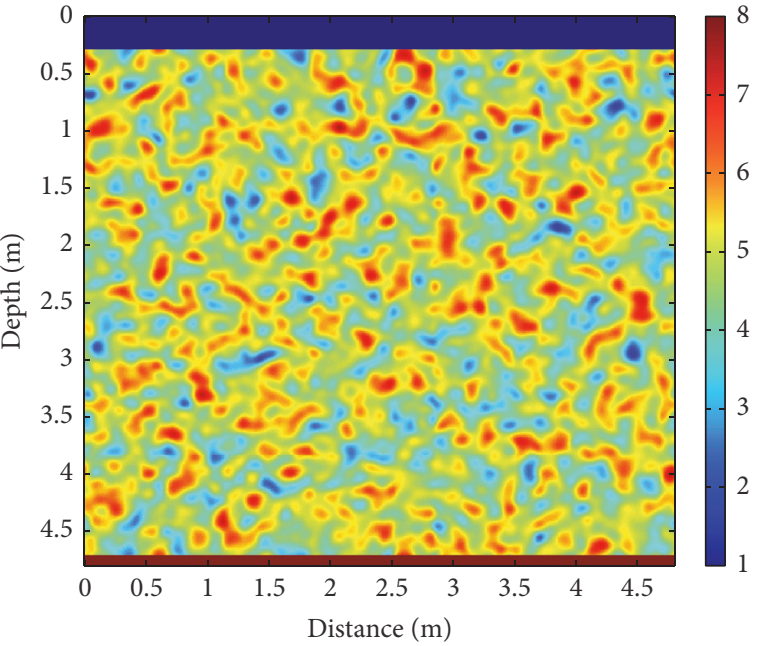

(b)

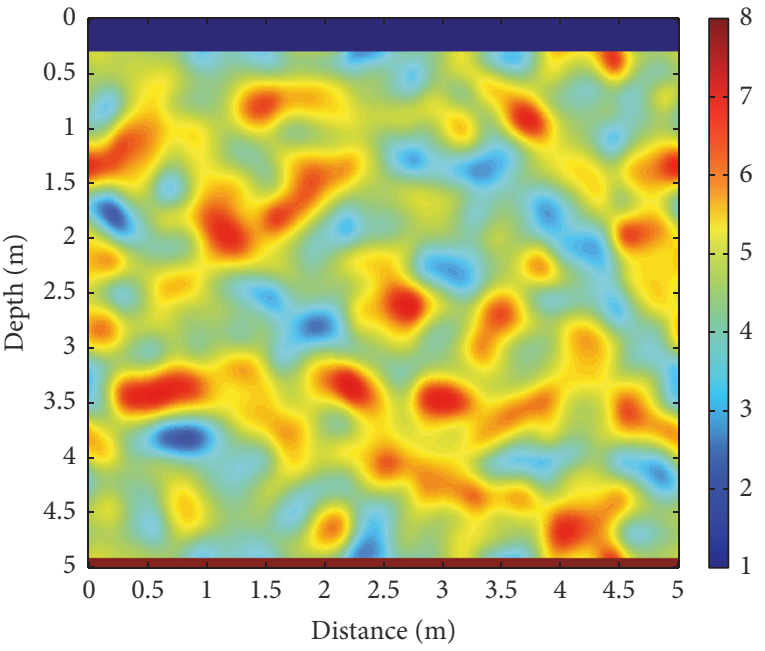

(d)

Figure 2: The discrete heterogeneous medium models, (a), (b), (c), and (d), with different autocorrelation lengths of $0.05 \mathrm{~m}, 0.1 \mathrm{~m}, 0.2 \mathrm{~m}$, and $0.3 \mathrm{~m}$, respectively.

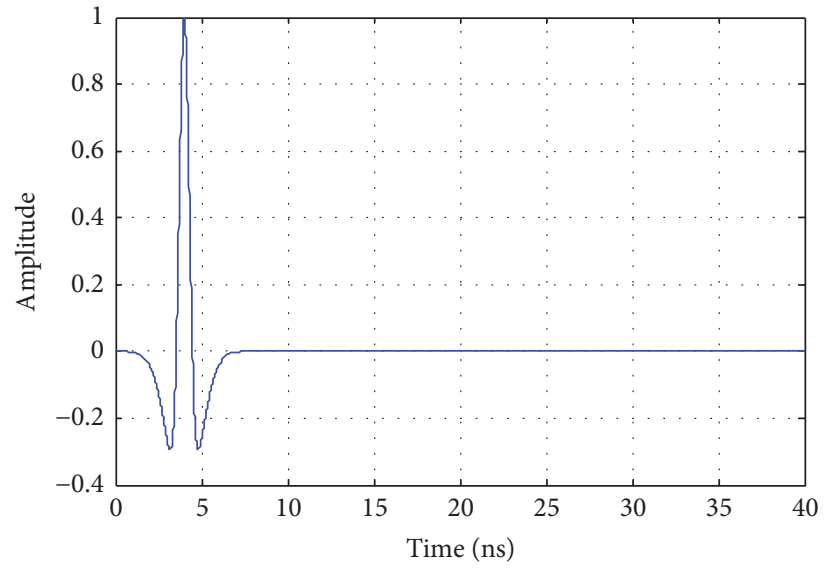

FIGURE 3: UWB Ricker pulse waveform. 
0.85 . The third layer serves as a reference layer with a permittivity of 8 and a depth of $0.1 \mathrm{~m}$. The autocorrelation length describes the scale of the random medium in the horizontal and vertical directions, which is manifested in the size of the rock fragments distributed randomly in the model, as shown in Figure 3. With an increase of the autocorrelation length, the size of the rocks increases and the number of rocks decreases. That is, the distribution of the real medium in the lunar regolith layer can be described effectively by selecting the appropriate parameters.

\section{The Numerical Simulation of LPR Channel 2}

4.1. The Selection of the Radiation Pulse Source. The radar radiation pulse source waveform is taken from [8], which can be calculated by UWB Ricker. Equation (8) [14] is defined as

$$
\begin{aligned}
& E_{\text {excit }}(\tau) \\
& =\frac{\phi_{\eta} \exp \left[-\pi^{2} \phi_{\eta}^{2}\left(\tau-\tau_{0}\right)^{2}\right]-\phi_{\lambda} \exp \left[-\pi^{2} \phi_{\lambda}^{2}\left(\tau-\tau_{0}\right)^{2}\right]}{\phi_{\eta}-\phi_{\lambda}} .
\end{aligned}
$$

We substituted the parameters of the LPR into (8), where $\phi_{\eta}$ $=750 \mathrm{MHz}, \phi_{\lambda}=250 \mathrm{MHz}$, and time $\tau_{0}$ is $4 \mathrm{~ns}$. Then, we can obtain the radiation pulse source waveform shown in Figure 3.

4.2. The Numerical Method of the 2D-FDTD. The differential Maxwell equations in the time domain are given by

$$
\begin{aligned}
& \nabla \times H=\varepsilon \frac{\partial E}{\partial t}+\sigma E \\
& \nabla \times E=-\mu \frac{\partial H}{\partial t}-\sigma H
\end{aligned}
$$

where $H$ is the magnetic field strength and $E$ is the electric field strength. $\varepsilon, \mu$, and $\sigma$ are the dielectric permittivity, permeability, and conductivity, respectively. Considering only the two-dimensional TM mode [15], all of the electric fields are transverse electric fields for the $y$ coordinates, and (9a) and (9b) contain only the directional components of $H_{x}, H_{z}$, and $E_{y}$. In the Cartesian coordinate system, the 2D-FDTD differential iterative equations in the TM mode are defined as

$$
\begin{aligned}
& H_{x}^{n+1 / 2}(i, j)=\frac{2 \mu_{x}(i, j)-\Delta t \sigma_{x}(i, j)}{2 \mu_{x}(i, j)+\Delta t \sigma_{x}(i, j)} \times H_{x}^{n-1 / 2}(i, j) \\
& +\frac{2 \Delta t}{2 \mu_{x}(i, j)+\Delta t \sigma_{x}(i, j)}\left(\frac{E_{y}^{n}(i, j+1)-E_{y}^{n}(i, j)}{\Delta z}\right), \\
& H_{z}^{n+1 / 2}(i, j)=\frac{2 \mu_{z}(i, j)-\Delta t \sigma_{z}(i, j)}{2 \mu_{z}(i, j)+\Delta t \sigma_{z}(i, j)} \times H_{z}^{n-1 / 2}(i, j) \\
& -\frac{2 \Delta t}{2 \mu_{z}(i, j)+\Delta t \sigma_{z}(i, j)}\left(\frac{E_{y}^{n}(i+1, j)-E_{y}^{n}(i, j)}{\Delta x}\right),
\end{aligned}
$$

$$
\begin{aligned}
& E_{y}^{n+1}(i, j)=\frac{2 \varepsilon_{y}(i, j)-\Delta t \sigma_{y}(i, j)}{2 \varepsilon_{y}(i, j)+\Delta t \sigma_{y}(i, j)} \times E_{y}^{n}(i, j) \\
& +\frac{2 \Delta t}{2 \varepsilon_{y}(i, j)+\Delta t \sigma_{y}(i, j)}\left(\frac{H_{x}^{n+1 / 2}(i, j)-H_{x}^{n+1 / 2}(i-1, j)}{\Delta z}\right. \\
& \left.\quad-\frac{H_{z}^{n+1 / 2}(i, j)-H_{z}^{n+1 / 2}(i, j-1)}{\Delta x}\right) .
\end{aligned}
$$

Moreover, in order to avoid the dispersion of the electromagnetic wave caused by the numerical calculation, it is necessary to satisfy the following conditional formula (see (11)) when the discrete interval parameters $\Delta t, \Delta x$, and $\Delta z$ approach zero.

$$
\Delta t \leq \sqrt{\frac{\mu \varepsilon}{\left(1 / \Delta x^{2}+1 / \Delta z^{2}\right)}} .
$$

4.3. Numerical Simulation of the Models. This section will make use of the 2D-FDTD numerical method to simulate the heterogeneous random medium models (a), (b), (c), and (d), which are established in Figure 2. As a comparison, the second layer in Figure 2(a) is replaced by homogeneous media with a permittivity of 3 and loss tangent of 0.005 . Before the simulation, we need to set the parameters of the simulation model. The transmitter and receiver are set in the same position as the point source. The simulation time window is set to $80 \mathrm{~ns}$. The discrete grid spacing is set to $0.01 \mathrm{~m}$. A data trace is detected with every movement of $0.043 \mathrm{~m}$ in the horizontal direction. The separation distance is in accordance with the real situation when the LPR probed the lunar surface. The simulated horizontal direction of the model is $5 \mathrm{~m}$, such that it receives 116 data traces. The discrete time step is set to $0.02 \mathrm{~ns}$, according to (11). Due to the limited memory of the computer, a PML (Perfectly Matched Layer) is used as the electromagnetic absorption boundary condition [16], which simulates the propagation of electromagnetic waves in free infinite space.

The simulation result of the model (a) was calculated by 2D-FDTD, shown as both the A-Scan and B-Scan in Figure 4. In the figure, the direct and coupling waves of the radargram are clearly shown, but the reflected echoes of the buried objects are not clearly displayed. The A-Scan waveforms were plotted from a trace of the B-Scan data, and it was found that the amplitudes of the direct and coupling wave reflected signals were much larger than those of the reflected signals from the lunar buried objects.

The main objective of this data processing is to analyze the reflection signal of the objects in the lunar regolith layer. For this reason, the amplitudes of the reflected signals of the direct and coupling waves can be suppressed by the threshold in (12) to relatively increase the amplitude of the reflection signal of the lunar buried objects.

$$
\begin{aligned}
& \text { Amplitude }(i, j) \\
& = \begin{cases}\text { threshold, } & \text { if } \mid \text { Amplitude }(i, j) \mid>\text { threshold, } \\
\text { Amplitude }(i, j), & \text { else. }\end{cases}
\end{aligned}
$$




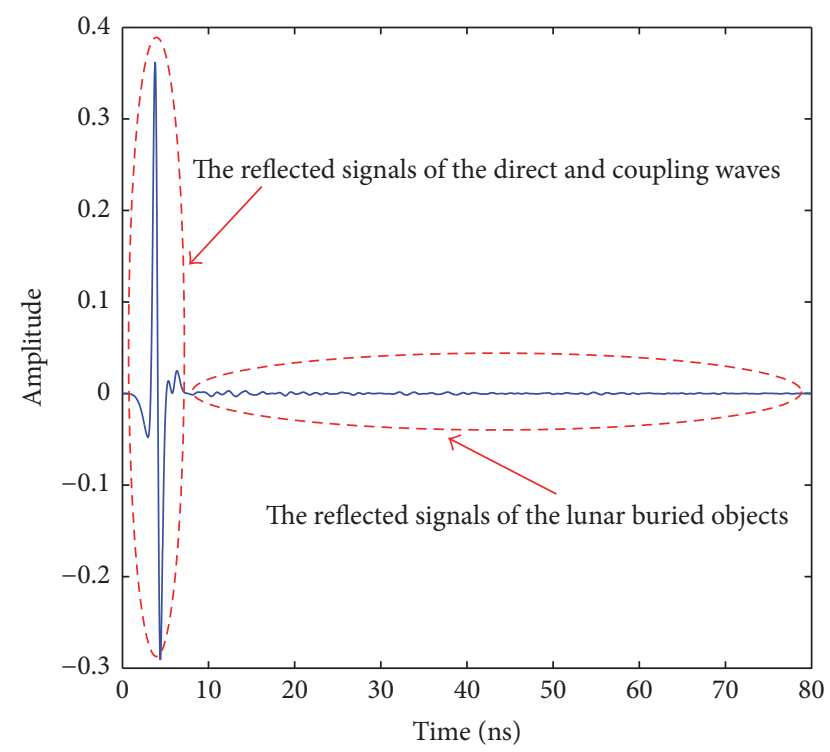

(a)

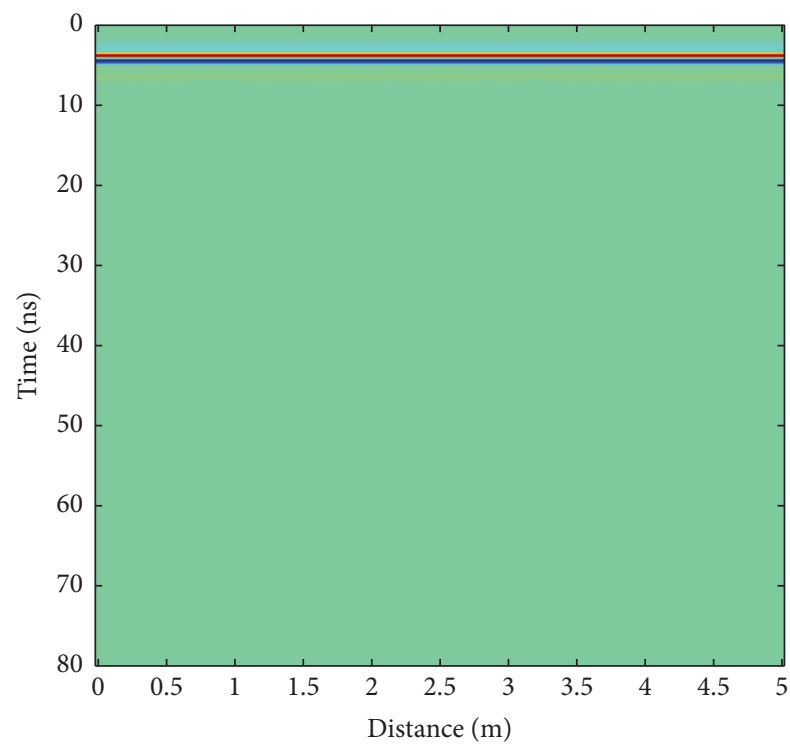

(b)

Figure 4: The simulation result of the random media model (a). The A-Scan of the simulated data is shown in (a). The B-Scan of the simulated data is shown in (b).

The amplitude threshold is set to 0.003 for each radar gram which are the simulation results of the models (a), (b), (c), and (d) and the homogeneous media. We plotted both the AScan and B-Scan simulation results, shown in Figure 5.

The time delay of the lunar surface is $7.91 \mathrm{~ns}$, as calculated in Figure 5. The reference layer echoes cannot be found from simulation results of models (a) and (b) because the radargrams are so cluttered, but they can be gradually found when they become more distinct, in models (c) and (d), from the homogeneous media, in which the time delays are $75.58 \mathrm{~ns}, 72.39 \mathrm{~ns}$, and $61.29 \mathrm{~ns}$, respectively. On the one hand, as the autocorrelation length decreases, the complexity of the model increases. The position of the echo signals of the reference layer is delayed because it is obscured by other signals and cannot be visually distinguished. On the other hand, when the autocorrelation length increases and the multiplied reflected echoes among the rock fragments are diminished, the amplitude of the reflected echoes of the buried targets in the lunar regolith layer model is gradually weakened, and the reference layer gradually becomes distinct. Moreover, this simulated experiment can also explain why the LPR did not find clear layers below the lunar surface at the Chang'E 3 landing site. The details of the investigation of the geological information of the lunar regolith layer will be elaborated in the following chapters.

\section{Comparison between the LPR Data and Simulated Data}

The LPR began work at 10:50:32 (UTC) on December 15, 2013, and ran until 14:16:56 (UTC) on January 15, 2014, when it stopped working due to a mechanical problem after a total of 277 minutes of work on the lunar surface [17]. Channel 2 received 2351 valid data traces during that working period. The probe distance is approximately $114 \mathrm{~m}$ along the path of the Yutu rover on the moon. Part of the LPR data at the landing site in the Mare Imbrium is shown in Figure 6. Meanwhile, we compared the LPR data with the simulated data calculated from the lunar regolith models by FDTD.

By comparing the LPR data and the simulation data, it is found that the echo characteristics of the two radargrams are similar. To quantify the degree of similarity of the two radargrams, the Bhattacharyya distance is used to analyze the two datasets in this paper. The method used to calculate the Bhattacharyya distance is defined as follows:

$$
D_{\text {dist }}\left(p_{\text {meas }}, q_{\text {simul }}\right)=-\ln \left(\sum \sqrt{p_{\text {meas }} \cdot q_{\text {simul }}}\right)
$$

where $p_{\text {meas }}$ and $q_{\text {simul }}$ are the LPR data and simulation data, respectively. The results of the Bhattacharyya distances of models (a), (b), (c), and (d) and the homogeneous model are $0.5829,0.7798,0.1165,0.1216$, and 0.1045 , respectively. It is clear that model (b) is the most similar to the LPR data among those results. This means that the heterogeneous random medium model (b) effectively corresponds to the interior structural characteristics of the lunar regolith. Hence, we can infer that the lunar regolith layer is not a purely regolith medium but rather has a distribution of a large number of rock fragments of uneven sizes and different shapes, and the diameters of the rock fragments are approximately $20 \mathrm{~cm}$. In addition, there are continuously reflected echo signals at $24 \mathrm{~ns}$, which may be a stratified structure in the lunar regolith layer or an echo signal of a continuous block of rocks. 

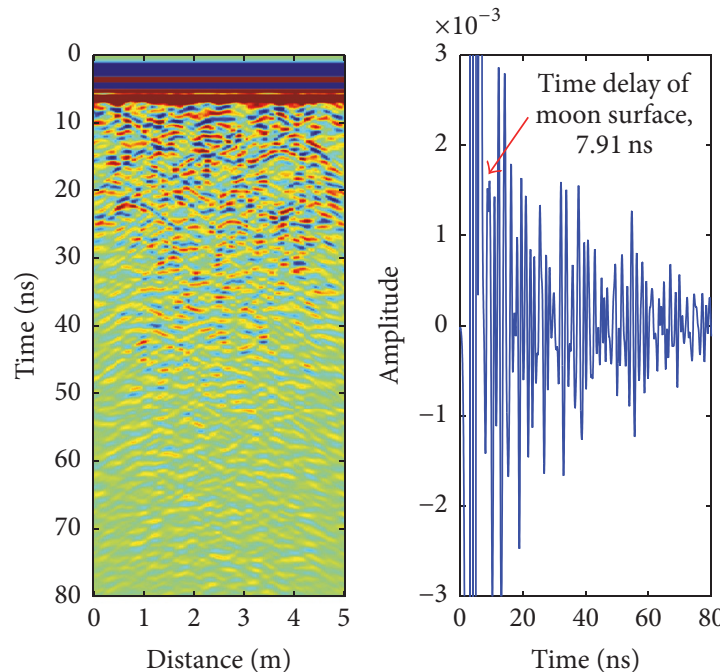

The simulated result of the model (a)
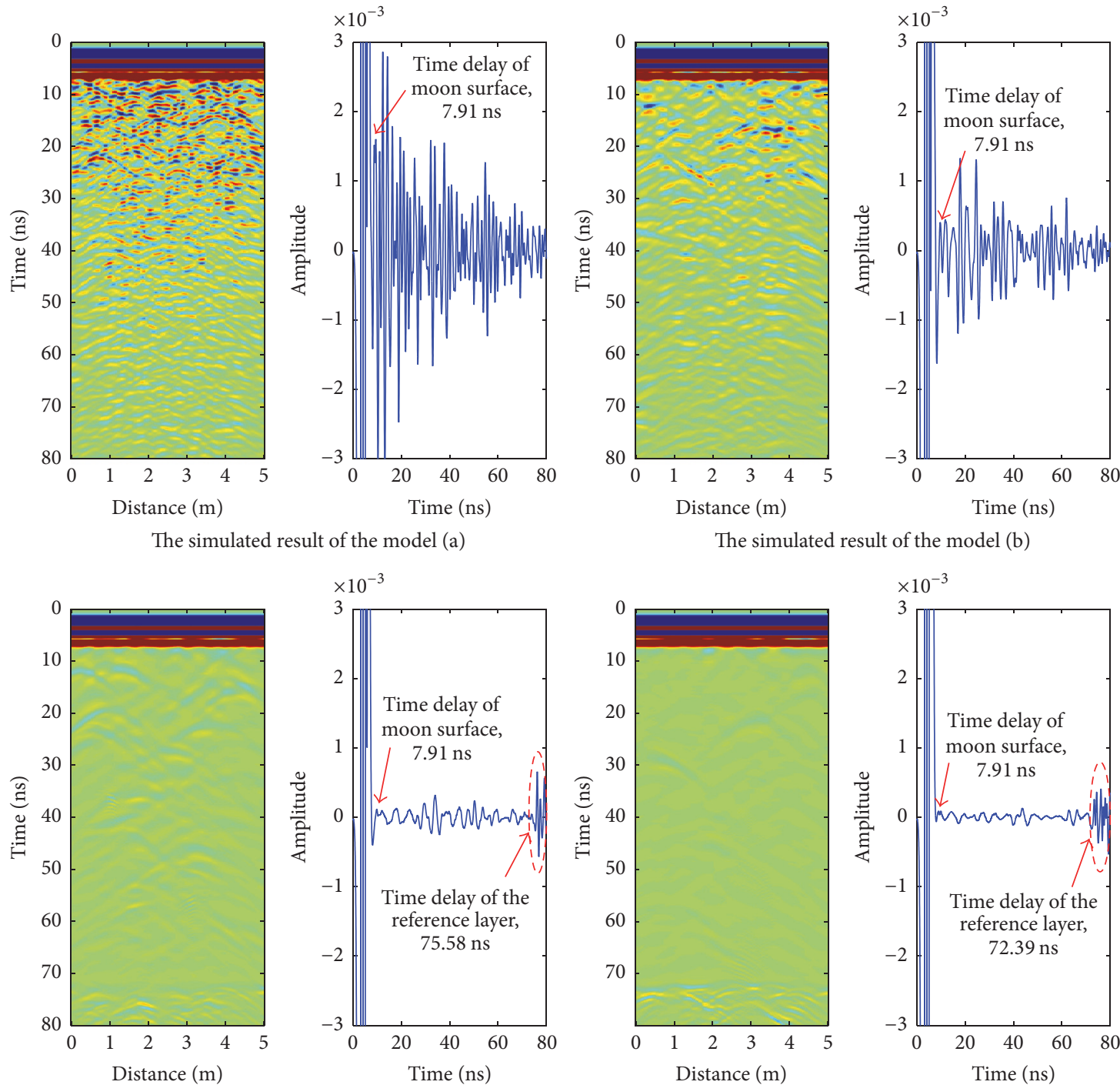

The simulated result of the model (b)
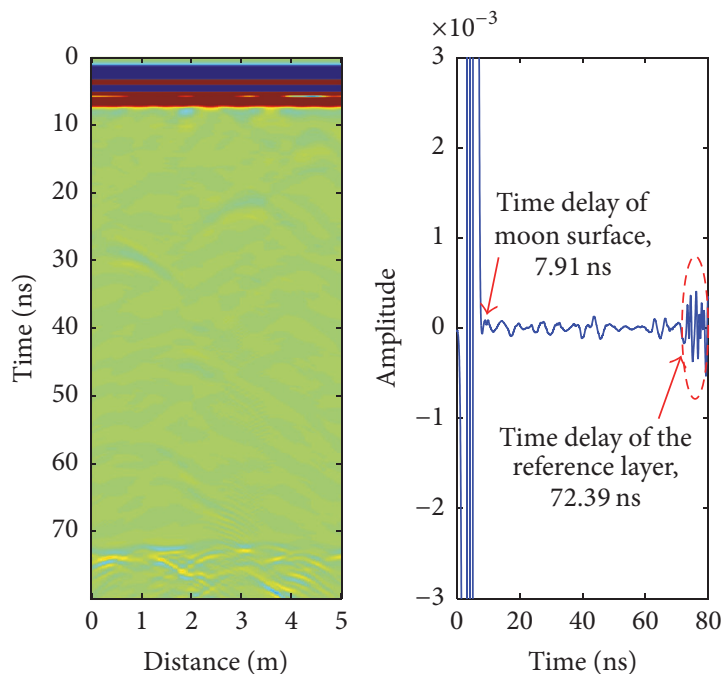

The simulated result of the model (c)

The simulated result of the model (d)
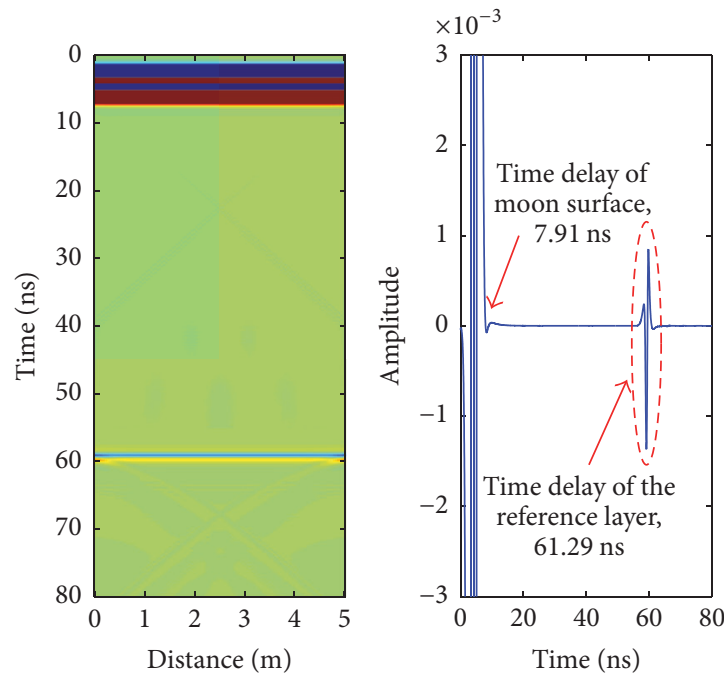

The simulated result of the homogeneous media model

Figure 5: The A-Scan and B-Scan simulation results of the models (a), (b), (c), and (d) and the homogeneous media with the amplitude threshold at 0.003 . 


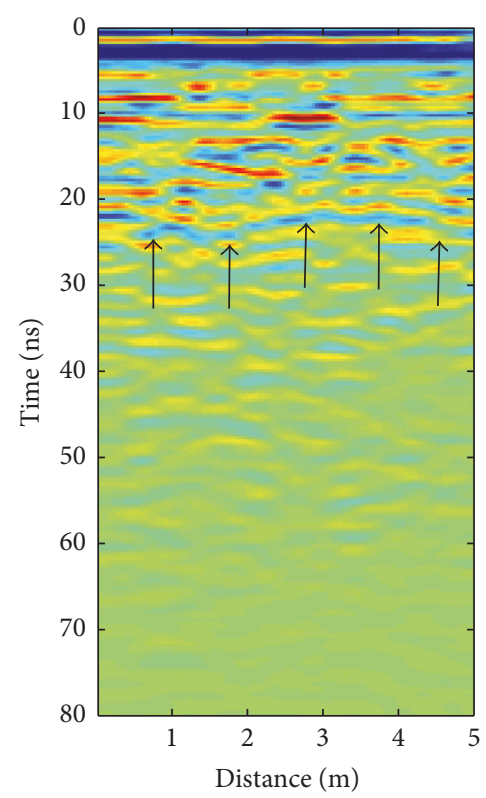

(a)

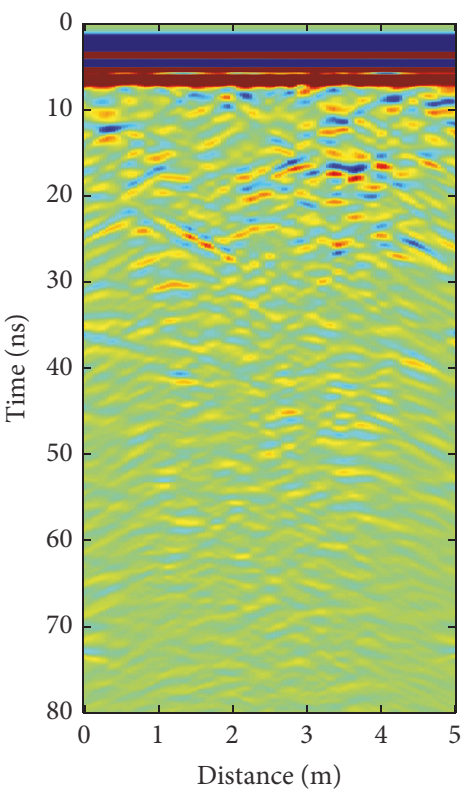

(b)

FIGURE 6: B-Scan of the LPR data (a) and the simulated data (b) of the heterogeneous random medium model (b).

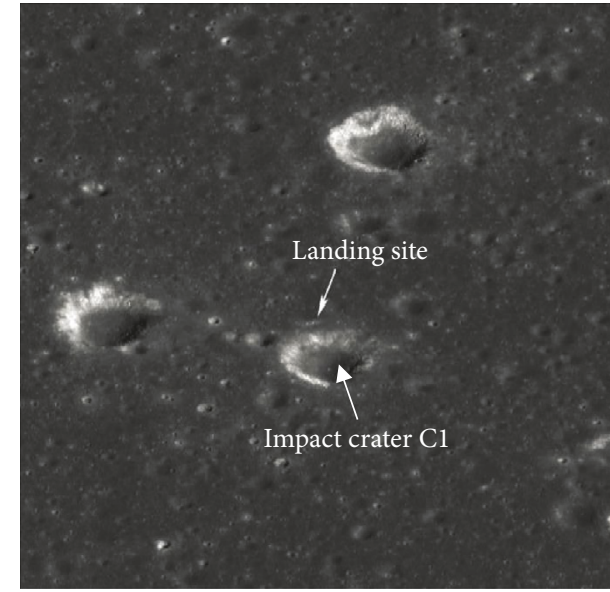

(a)

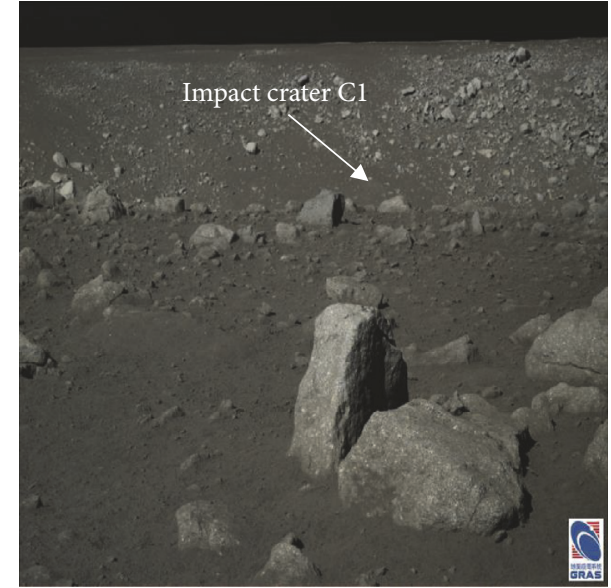

(b)

FIgURE 7: Chang'E 3 landing site and the topography of the impact crater C1. (a) is from Arizona State University, and (b) is from the Science and Application Center for Moon and Deep Space Exploration.

\section{The Interior Structure of the Lunar Regolith at the Chang'E 3 Landing Site}

The Chang'E 3 landed near the young crater $\mathrm{Cl}$, which has a diameter of approximately $450 \mathrm{~m}[18,19]$. The landing position was approximately $50 \mathrm{~m}$ from the edge of the impact crater. It can be seen in Figures 1 and 7 that the lunar surface is scattered with a large number of rock fragments of different sizes. At the impact edge, the distribution of rock fragments is even denser. These rocks originate from the lunar crater formation process. When the meteorite crashed on the lunar surface, the bedrock was contacted, squeezed, and crushed. This process formed an ejecta blanket and dug out material from deeper sections of the lunar surface. Some rocks were gasified or melted by the high temperatures, forming new material. The broken rock fragments were also formed because the meteorite impact process involves a massive transfer of mechanical energy to heat energy. Therefore, the large-scale materials sputtering over the original lunar regolith layer formed an ejecta layer, which could also be called a new lunar regolith layer, including a large number of new materials formed by the high temperature, such as impact breccias, glass, and metal, and broken rock blocks.

Xiao et al. [18] used a diameter-frequency method to estimate the geological age of the landing site (impact crater C1) at a minimum model age of 27 million years (My) and a 


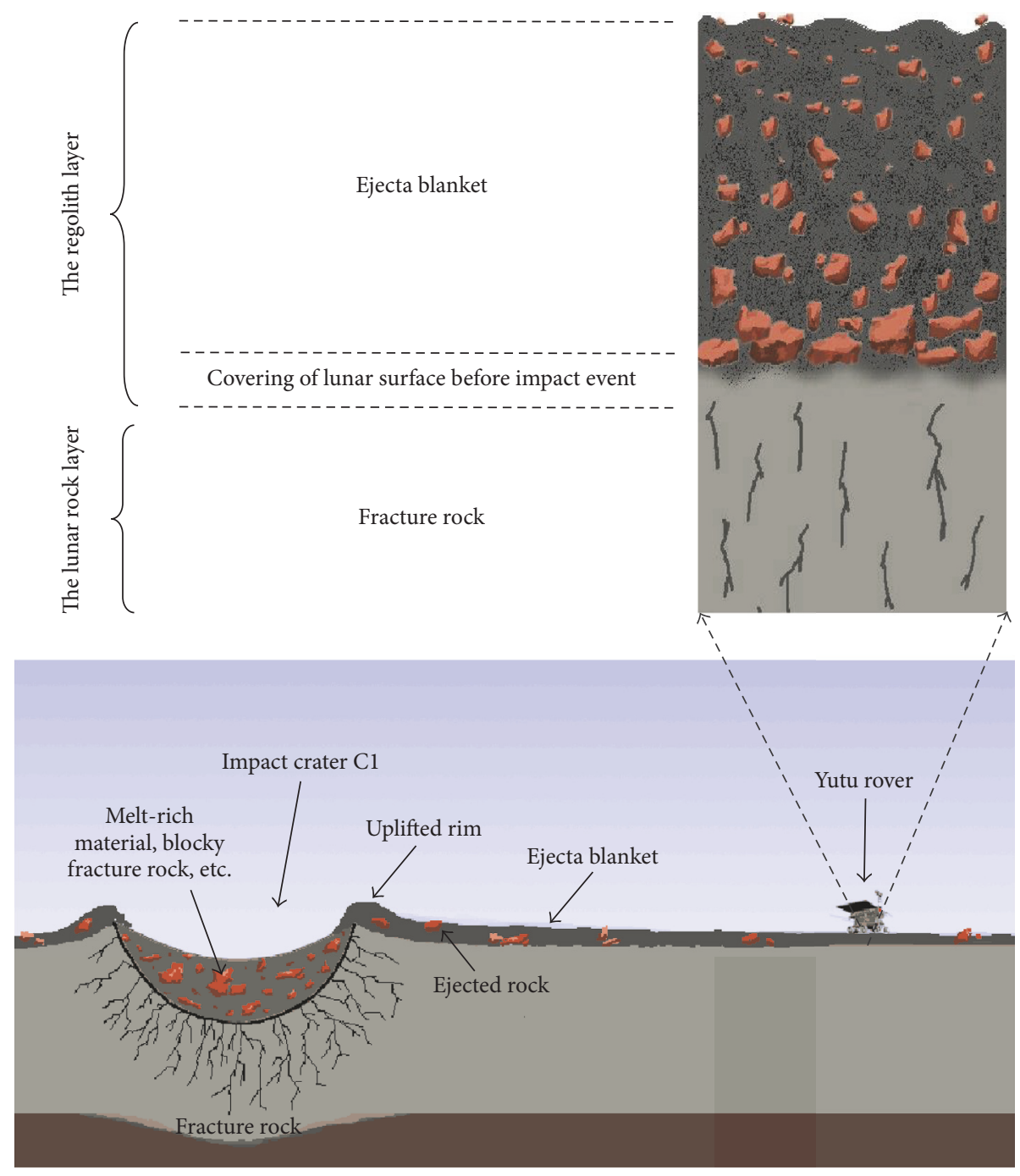

FIGURE 8: The diagram of the interior structure of the lunar regolith layer at the Chang'E 3 landing site.

maximum model age of 80 million years (My). This indicates that the geological structure is very young, the lunar regolith is immature, and the internal structure is rock-like. Basilevsky et al. [20] compared the photographs taken by the Lunokhod and Yutu rovers, which indicated that there exist rock blocks with diameters of dozens of centimeters across the lunar surface. In other words, it can be inferred that the interior of the lunar regolith also contains similar scales of rocks. This is consistent with one of the conclusions of the current study, namely, that the interior of the lunar regolith is a regolithrock mixture.

Based on the above studies, the subsurface geological structures of the Chang'E 3 landing site are probably divided into a lunar regolith layer and a lunar rock layer, as shown in Figure 8. The lunar regolith layer includes the materials of the ejecta blanket and the original lunar regolith layer. We did not include the lunar dust layer because the boundary between the lunar dust layer and lunar regolith layer is difficult to define in this dataset. Because the geological structure of the Chang'E 3 landing site is very young, with immature lunar regolith and more internal stones, it is easy to misidentify the continuous echoes produced by a number of adjacent lunar rocks as a layered structure in the radargram. According to the statistics of the Apollo samples, as the sampling depth increases, the average diameter of the rocks in the lunar regolith layer increases slightly. In general, lunar rock size is related to maturity. The higher the maturity level, the smaller the diameter of lunar rocks. The maturity is related to the time of exposure on lunar surface [21]. It can be seen that the maturity of the lunar regolith is low at the landing site; the distribution of the interior rocks is irregular, and the geological structure is young at the Chang'E 3 landing site. Therefore, one possible structure is a fractured rock layer that transitions to the bedrock layer below the lunar regolith layer.

The specific depth of the lunar regolith layer is not determined because the simulation results show that, within a certain range, as the number of rocks increases, the electromagnetic wave propagation in that medium will produce multiple reflections and scattering, which makes the radar echoes too complicated. Thus, an accurate depth of the lunar regolith 
is difficult to determine by LPR. In fact, the boundary between the lunar regolith layer and the lunar rock layer is not clear [22]. Rather, it is a gradual structure from top to bottom, which makes it difficult for radar to distinguish interior layers.

\section{Conclusion and Discussion}

Based on the theory of the random medium model, the Apollo drilling samples data, and the real lunar surface at the Chang'E 3 landing site, the model of a heterogeneous random medium is established. By using the FDTD numerical method, electromagnetic wave propagation is simulated and the resulting radar echoes are obtained. Comparing the LPR data and the simulated data, the following conclusions are obtained:

(1) The lunar model of a heterogeneous random medium is more consistent with the real structure of the lunar regolith than other theoretical models of the lunar regolith layer used in the preceding literature.

(2) The radar echoes become more complicated as the number of rocks increases, within a certain range. Thus, the accurate depth and interior structure of the lunar regolith are difficult to determine by LPR.

(3) The interior of the lunar regolith is not a purely uniform medium but has a distribution of regolith-rock mixture media with different rock sizes and shapes. The diameter of the rock fragments is approximately $20 \mathrm{~cm}$ in the lunar regolith layer, which is larger than the $4 \mathrm{~cm}$ diameter of the sample from the Apollo mission.

(4) The site produces clear layered echoes at approximately $24 \mathrm{~ns}$ in the LPR data, shown in Figure 6. These layered echoes can be interpreted as stratified structures in the interior of the lunar regolith or as a large number of small-scale lunar rocks that produce overlapping radar echo signals.

The future Chang'E 5 program will be equipped with a lunar regolith radar sounder, which will have a higher detection resolution and working frequency than the LPR. Therefore, further studies of the application of the heterogeneous random medium method in lunar regolith modeling will be helpful to interpret the lunar radar data and better understand the real distribution characteristics and structures of the lunar regolith. In addition, the traditional homogeneous multireflection filtering method [23] has been difficult to use to filter the multiple reflection electromagnetic waves of the radar echoes in the lunar regolith layer. Using statistical methods, it is possible to establish a multireflection filtering method based on the random medium theory, which may be a breakthrough for solving complicated filtering problems.

\section{Conflicts of Interest}

The authors declare that they have no conflicts of interest.

\section{Acknowledgments}

The authors thank the Ground Application System of Lunar Exploration, NAOC, for providing the lunar penetrating radar data. This work was supported by NAOC through Grant no. Y734061V01 and the National Natural Science Foundation of China (Grant no. 41403054).

\section{References}

[1] Z. Y. Ouyang, Introduction of Lunar Sciences, China Aerospace Publishing House, Beijing, China, 2005.

[2] O. E. Berg, F. F. Richardson, and H. Burton, "Apollo 17 preliminary science report," Tech. Rep. 16, NASA SP-330, 1973.

[3] Y. G. Shkuratov and N. V. Bondarenko, "Regolith layer thickness mapping of the moon by radar and optical data," Icarus, vol. 149, no. 2, pp. 329-338, 2001.

[4] A.-L. Lan and S.-W. Zhang, "Study on the thickness of lunar regolith with microwave radiometer," Romote Sensing Techoeslogy And Application, vol. 03, pp. 154-158, 2004.

[5] W. Fa and Jin Y., "Simulation of Multiple-Channels radiance brightness temperature on lunar surface and inversion of lunar regolith layer thickness," Progress in Natural Science, vol. 01, pp. 86-94, 2006.

[6] Z.-G. Meng, S.-B. Chen, and C. Liu et al., "Simulation on passive microwave radiative transfer in inhomogeneous lunar regolith," Journal of Jinlin University (Earth Science Edition), vol. 06, pp. 1070-1074, 2008.

[7] S. Chen, F. Zha, and Y. Lian et al., "Forward modelling of the lunar regolith layer using radar detection based on FDTD," Earth Science Frontiers, vol. 21, no. 6, pp. 88-91, 2014.

[8] G.-Y. Fang, B. Zhou, Y.-C. Ji et al., "Lunar penetrating radar onboard the Change-3 mission," Research in Astronomy and Astrophysics, vol. 14, no. 12, pp. 1607-1622, 2014.

[9] L. Ikelle, S. Yung, and F. Daube, "2-D random media with ellipsoidal autocorrelation functions," Geophysics, vol. 58, no. 9, pp. 1359-1372, 1993.

[10] S. C. N. Ergintav, "Modeling of multi-scale media in discrete form," Journal of Seismic Exploration, vol. 6, no. 1, pp. 77-96, 1997.

[11] X. Xi and Y. Yao, "Simulations of random medium model and intermixed random medium," Earth Science-Journal of China University of Geosciences, vol. 27, no. 1, pp. 67-71, 2002.

[12] Z. Jiang, Z. Zeng, J. Li, F. Liu, and W. Li, "Simulation and analysis of GPR signal based on stochastic media model with an ellipsoidal autocorrelation function," Journal of Applied Geophysics, vol. 99, pp. 91-97, 2013.

[13] G. H. Heiken, Vaniman D. T., and B. M. French, Lunar Source-Book: A Users Guide to the Moon, New York, NY, USA Cambridge University Press, 1999.

[14] S. Yu, "Wide-band Ricker wavelet," OGP, vol. 31, no. 05, pp. 605615, 1996.

[15] A. Z. Elsherbeni, The Finite-Difference Time-Domain Method for Electromagnetics with MATLAB Simulations, SciTec Publishing, Inc, Raleigh, NC, USA, 2006, 2-32.

[16] J.-P. Berenger, "A perfectly matched layer for the absorption of electromagnetic waves," Journal of Computational Physics, vol. 114, no. 2, pp. 185-200, 1994.

[17] Y. Su, G.-Y. Fang, J.-Q. Feng et al., "Data processing and initial results of Chang'e-3 lunar penetrating radar," Research in Astronomy and Astrophysics, vol. 14, no. 12, pp. 1623-1632, 2014. 
[18] L. Xiao, P. Zhu, G. Fang et al., "A young multilayered terrane of the northern Mare Imbrium revealed by Chang'E-3 mission," Science, vol. 347, no. 6227, pp. 1226-1229, 2015.

[19] J. Zhang, W. Yang, S. Hu et al., "Volcanic history of the Imbrium basin: a close-up view from the lunar rover Yutu," Proceedings of the National Academy of Sciences of the United States of America, vol. 112, no. 17, pp. 5342-5347, 2015.

[20] A. T. Basilevsky, A. M. Abdrakhimov, J. W. Head, C. M. Pieters, Y. Wu, and L. Xiao, "Geologic characteristics of the Luna 17/Lunokhod 1 and Chang'E-3/Yutu landing sites, Northwest Mare Imbrium of the Moon," Planetary and Space Science, vol. 117, pp. 385-400, 2015.

[21] B. B. Wilcox, M. S. Robinson, P. C. Thomas, and B. R. Hawke, "Constraints on the depth and variability of the lunar regolith," Meteoritics and Planetary Science, vol. 40, no. 5, pp. 695-710, 2005.

[22] J. Feng, Y. Su, and C. Ding et al., "Dielectric properties estimation of the lunar regolith at CE-3 landing site using lunar penetrating radar data," Icarus, vol. 284, pp. 424-430, 2017.

[23] S. Caorsi and M. Stasolla, "GPR data enhancement via multiple reflections' filtering," International Journal of $R F$ and Microwave Computer-Aided Engineering, vol. 23, no. 4, pp. 417-422, 2013. 


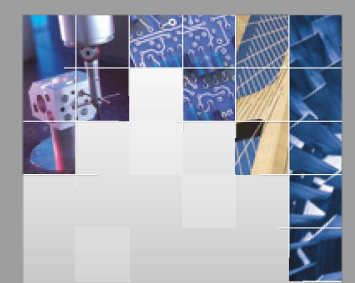

\section{Enfincering}
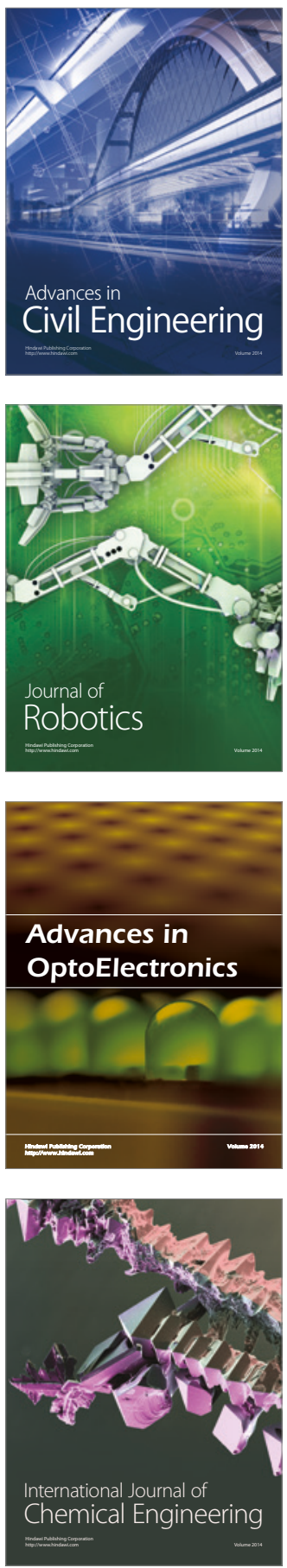

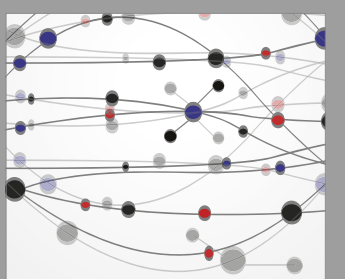

The Scientific World Journal

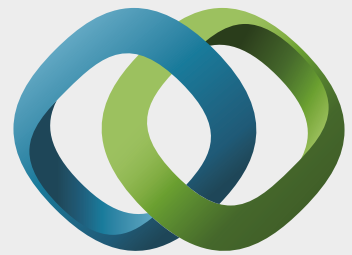

\section{Hindawi}

Submit your manuscripts at

https://www.hindawi.com
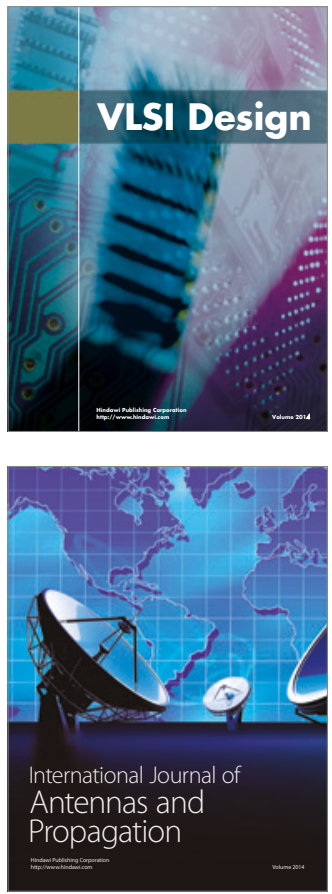

\section{Rotating}

Machinery
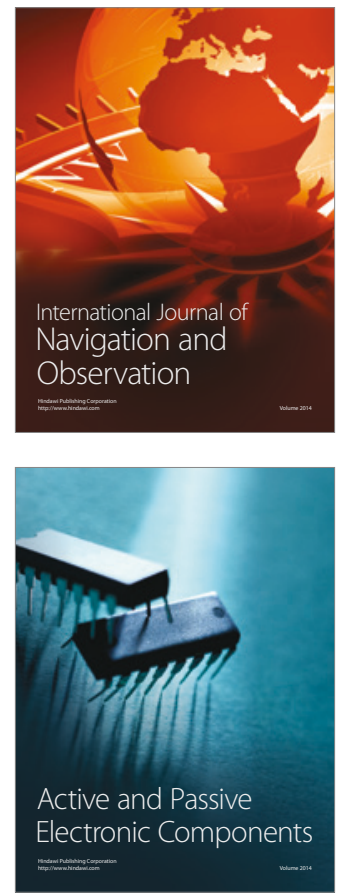
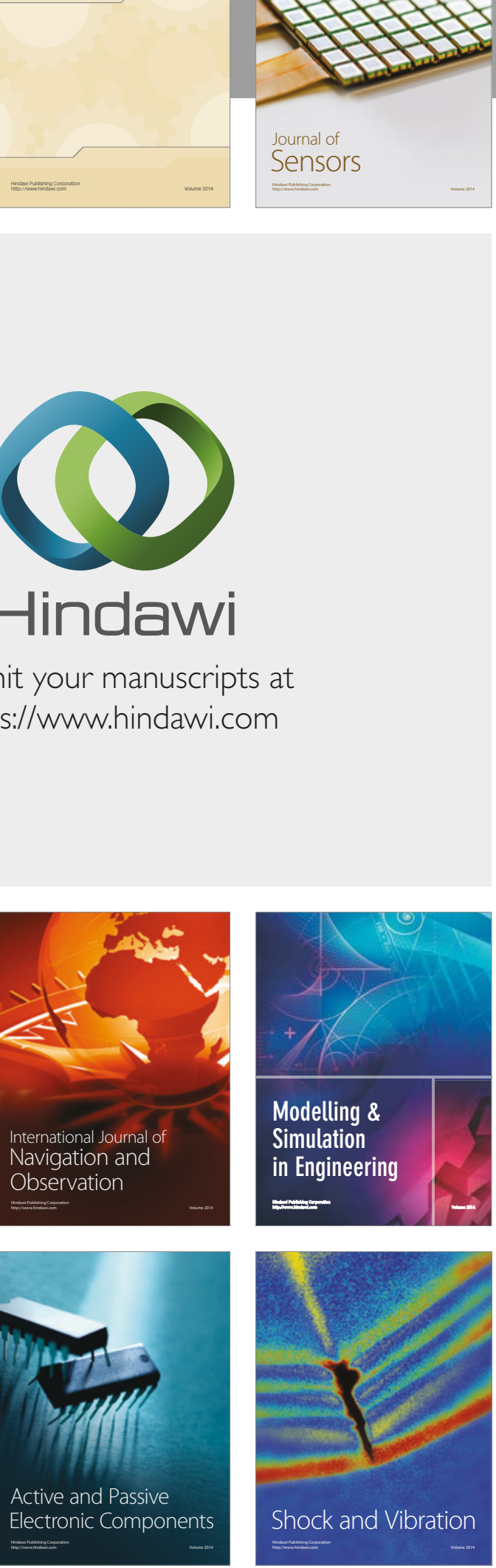
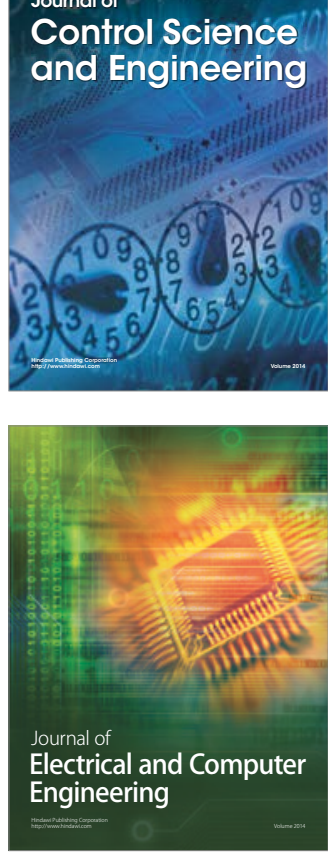

Distributed

Journal of

Control Science

and Engineering
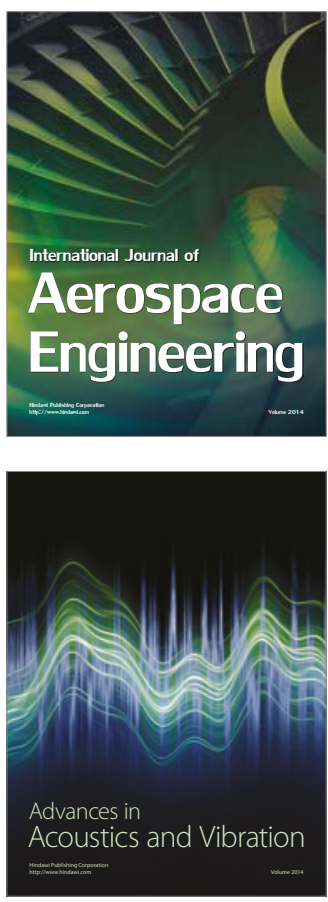

Sensor Networks 